\title{
Análisis de datos sobre el uso y consumo de servicios internet diferenciado por género en estudiantes universitarios
}

\author{
Analysis of data on the use and consumption of internet \\ services differentiated by gender in university students
}

\author{
Carmen Burgos Videla ${ }^{1 *} \quad$ Wilson Castillo-Rojas ${ }^{2}$ \\ Recibido: 6 de Enero de 2021, aceptado 30 de Abril de 2021 \\ Received: January 6, $2021 \quad$ Accepted: April 30, 2021
}

\begin{abstract}
RESUMEN
El estudio tiene como objetivo obtener una descripción diferenciada por género en un contexto universitario, acerca del uso y consumo de aplicaciones y/o servicios a través de Internet. El trabajo de análisis se aborda desde una metodología mixta con análisis descriptivo y cuantitativo. En primer lugar, se utilizan técnicas estadísticas para el análisis exploratorio de los datos, y posteriormente se generan modelos de clustering que permiten complementar la descripción del conjunto de datos sobre el objeto de estudio. Para esto, se toma como base el resultado de la encuesta basada en la primera dimensión del instrumento llamado Competencias Básicas Digitales (COBADI), en los aspectos preliminares que tienen relación con: tenencia de las TICs necesarias, el lugar y tipo de conexión, así como el uso y consumo de aplicaciones y/o servicios, para acceder a través de Internet. Los resultados muestran que tanto mujeres y hombres orientan el uso y consumo de las TICs, de manera diferente para sus actividades tanto personales como académicas. El estudio logra concluir que este análisis por género, permite establecer una línea base para poder profundizar posteriormente, en el desarrollo de las competencias digitales necesarias actualmente, que deben desarrollar estudiantes universitarios.
\end{abstract}

Palabras claves: Tecnologías de la información y comunicación, competencia digital, consumo de tecnología, análisis de datos.

\begin{abstract}
The study aims to obtain a description differentiated by gender in a university context, about the use and consumption of applications and/or services through the Internet. The analysis work is approached from a mixed methodology with descriptive and quantitative analysis. Firstly, statistical techniques are used for the exploratory analysis of the data, and later clustering models are generated that complement the description of the data set about the object of study. For this, the result of the survey based on the first dimension of the instrument called Basic Digital Competences (COBADI) is taken as a basis, in the preliminary aspects that are related to: the possession of the necessary ICTs, the place and type of connection, as well as the use and consumption of applications and/or services, to access via the Internet. The results show that both women and men guide the use and consumption of ICTs, differently for their personal and academic activities. The study concludes that this analysis by gender allows establishing a baseline to be able to delve further, in the development of the digital skills currently required, and which should be developed by university students.
\end{abstract}

Keywords: Information and communication technologies, digital competence, technology consumption, data analysis.

1 Universidad de Atacama. Instituto de Investigación en Ciencias Sociales y Educación (IICSE). Copiapó, Chile. E-mail: carmen.burgos@uda.cl

2 Universidad de Atacama. Departamento de Ing. en Informáticas y Ciencias de la Computación, Instituto de Investigación en Ciencias Sociales y Educación (IICSE). Copiapó, Chile. E-mail: wilson.castillo@uda.cl

* Autor de correspondencia: carmen.burgos@uda.cl 


\section{INTRODUCCIÓN}

Las competencias, como constructo incorporado al discurso educativo en los noventa son definidas desde un paradigma tecnológico, convirtiéndose en la punta de lanza de las innovaciones curriculares y en determinar un valor agregado a los sujetos que las manejan, como posibilidad de progreso social e individual en el mundo globalizado. Por tanto, esta posición se concreta en los currículos de cualquier nivel educativo; las competencias en relación con los tres ámbitos de esta, atienden a las maneras por las cuales los sujetos demuestran que el contenido, la dimensión teórica del conocimiento es entendido y aprehendido, expresándose procedimentalmente $\mathrm{y}$ actitudinalmente.

En ese sentido, las competencias en cualquier ámbito disciplinar son entendidas como un proceso en el cual las personas pueden resolver creativamente problemas, realizar actividades, formular preguntas, buscar información relevante, analizar, comprender $\mathrm{y}$ reflexionar al aplicar su conocimiento, dando una respuesta a las demandas de un entorno real [1].

Lo anterior, sumado al avance de la ciencia y la tecnología, ha obligado a adaptar la forma de vivir y actuar en muchos ámbitos, dentro de ellos, la educación, donde se espera que los docentes apliquen métodos y herramientas nuevas, generando procesos de innovación. Los docentes y en general los sujetos deben adquirir competencias para adaptarse a las maneras de aprender de las nuevas generaciones donde la mayoría son nativos digitales. Para no ser parte de esta obsolescencia tecnológica, deben existir constantes capacitaciones para los profesionales de la educación, y la preparación adecuada para los estudiantes.

Dentro de este enfoque, los individuos deben ser capaces no solo de utilizar las nuevas tecnologías, sino que capaces de solucionar las problemáticas que se le presenten. Y así establece la UNESCO, en su texto ICT Competency Framework for Teachers [2], señalando que la nueva sociedad del conocimiento busca fomentar la diversidad y compartir estas nuevas formas de obtener el conocimiento, englobando todas las comunidades que son parte de cada sociedad. Todas las personas son capaces de adquirir información y además transformarla en conocimiento, para contribuir al desarrollo económico y social de su comunidad.
Bajo este contexto, el estudio que se presenta en este artículo tiene como objetivo obtener una descripción por género, acerca del consumo y utilización de las TICs por parte de estudiantes dentro de un contexto universitario del siglo XXI. Específicamente, se trata de contrastar y diferenciar por género, el uso cotidiano de las TICs, en todos aquellos servicios de Internet que los estudiantes utilizan para sus actividades tanto de: ocio, comunicativas, de interacción, y de trabajo académico, los cuales les permiten desarrollar sus competencias digitales.

Para esto, el estudio se enfoca en los aspectos básicos del instrumento COBADI, que tienen relación, en primer lugar, con la tenencia o de contar con la tecnología base para poder utilizar servicios de Internet, como por ejemplo si cuenta con: computador, tablet, móvil y otros, así como si dispone de una conexión apropiada. En segundo lugar, aspectos relacionados con el consumo de servicios de Internet, para llevar a cabos sus actividades dentro de un contexto universitario.

En el trabajo de análisis, en principio se utilizan métodos estadísticos para el análisis exploratorio de los datos, y posteriormente técnicas de clustering. Esto último, para describir por género los hábitos de uso y consumo en los servicios/aplicaciones de Internet, así también, como mecanismo de validación de los resultados preliminares.

Este estudio permitirá establecer la línea base para un análisis posterior más profundo, acerca de las competencias digitales que logran desarrollar los estudiantes, con la tecnología necesaria para sobrellevar sus actividades, tanto personales como académicas, y el hábito regular de utilizar la tecnología para propósitos de aprendizaje.

\section{TRABAJOS RELACIONADOS}

El concepto de competencias digitales ha sido ampliamente investigado, y se entienden como "instrumentos de gran utilidad que permite la movilización de actitudes, conocimientos y procesos; por medio de los cuales los discentes adquieren habilidades para facilitar la transferencia de conocimientos y generar innovación" [3]. Este uso de instrumentos no debe ser limitado solo al accionar, sino también el transmitir los conocimientos deseados en una variedad de situaciones, para lo 
cual los docentes y estudiantes deben ser flexibles y comprensivos [4]. La UNESCO declaró también que las Tecnologías de la Información y las Comunicaciones (TICs), deben ser aprovechadas para fortalecer los sistemas educativos, apoyando así la difusión del conocimiento, el acceso a la información, asegurando calidad y promoviendo el aprendizaje efectivo [1].

La necesidad de implementación de nuevas tecnologías en lo educativo abre brechas que deben ser atendidas, puesto que los docentes deben manejar una competencia determinada y transferirla a los estudiantes [5]. Se debe tener en cuenta que no solo basta con el acceso a las herramientas, sino considerar la alfabetización digital en las intervenciones educativas, necesarias para promover la igualdad de oportunidades en la sociedad [6].

El proceso de alfabetización digital, según Calle y Lozano [7], conlleva conseguir ciertas competencias que faciliten la construcción de un sujeto que es capaz de interactuar a través de las TICs. Uno de los reportes de la OCDE sobre el impacto de la era digital en el bienestar de las personas señala que la tecnología puede tener tanto un impacto positivo como negativo sobre la vida de las personas [8].

El informe OCDE del 2019, muestra que en Chile faltan generar estadísticas respecto al uso de las tecnologías. Hay alta exposición a riesgos y bajo aprovechamiento de oportunidades en el uso de la tecnología. El acceso a internet está sobre el promedio OCDE, en $87,5 \%$, es el segundo país con uso de internet menos variado y la inequidad en los usos está sobre el promedio OCDE. Una de las principales desventajas y riesgo refieren a las bajas habilidades digitales, poco uso de educación on-line, alto nivel de trabajos en riesgo de automatización, y ocupan el primer lugar en uso extremo de internet entre jóvenes de 15 años.

Es importante constatar que las habilidades TICs están presentes en los Estándares para la Formación Inicial Docente de Educación Parvularia, Básica y Media, se presentan como parte de una de las ocho habilidades profesionales y como parte del estándar relacionado con diseño de estrategias. Sin embargo, existen desafíos en la implementación y evaluación del desarrollo de la formación en esta área, para asegurar competencias actualizadas en el uso y consumo de tecnología.

La sociedad hoy en día enfatiza en la adquisición de competencias digitales, éstas son las que incluyen las TICs, pero la falta de profundización en la determinación de ciertos conceptos asociados a competencias digitales, disminuye la incorporación de las nuevas tecnologías en el quehacer docente. $\mathrm{La}$ Competencia Digital es una de las ocho competencias que la Unión Europea establece como clave en sus estrategias de aprendizaje permanente [9].

Díaz y Serra (2020), señalan que existe una necesidad de identificar fortalezas y debilidades de los docentes en el uso de TICs para generar cambios en su incorporación al ejercicio profesional, para lo cual, presentan un estudio contextualizado en una universidad venezolana, donde no se ha contemplado el tema de la revisión objetiva o subjetiva de las competencias digitales de los docentes, y que se necesita que los docentes sean capaces de realizar cambios positivos para solucionar problemas con el uso de nuevas tecnologías implementadas en el aula. Lévano-Francia et al. [5], destacan que los rápidos cambios introducidos por las nuevas tecnologías emergentes, obligan a la sociedad a adaptarse a ellas para su uso en la vida cotidiana y profesional. En el ámbito educativo esto está convirtiéndose en casi una necesidad, donde la obsolescencia no tiene cabida y se deben realizar esfuerzos para la alfabetización digital de los profesionales de la educación. Debiese existir en la actualidad una preparación adecuada en la formación docente respecto a competencias digitales, las cuales permitan un manejo de las herramientas básicas que den paso a mejoras en el aprendizaje. Las instituciones educativas no pueden quedar al margen de los avances tecnológicos de la actualidad.

Gutiérrez, Guzmán y Jiménez [10], señalan que, dentro de los primeros estudios y comparaciones realizadas respecto al uso de nuevas tecnologías en el año 1995 por la Unesco, se observaba una gran brecha, y las mujeres poseían bajo manejo de las TICs, las cuales se consideran una herramienta indispensable para el progreso de la igualdad y democracia. Conforme el avance de los años, las mujeres han demostrado un gran avance respecto al uso y manejo de estas tecnologías, y comenzaron a estudiarse en: la primera brecha de género (acceso), 
la segunda brecha de género (uso), y la más reciente, una tercera brecha (apropiación).

La segunda brecha, es la que presenta mayor desventaja de género (intensidad, tipo de uso y competencias digitales), donde se evidencia que las mujeres realizan mayoritariamente acciones que no requieran una gran destreza tecnológica, como usar un chat o el correo electrónico.

En la tercera brecha, se ha documentado que la distancia entre número de mujeres y hombres que se dedican a trabajar en TICs, es de 3 a 7 en países europeos. En el estudio de caso múltiple realizado de Gutiérrez, Guzmán y Jiménez [10], se evidencia que si bien, los docentes conocen distintas herramientas tecnológicas, sólo el $26 \%$ implementa el uso de la plataforma educativa digital disponible en la universidad en la cual se basó la muestra; y que el mayor obstáculo para la implementación de nuevas herramientas digitales es la falta de tiempo en la jornada laboral y del hogar, una realidad evidente para todos los docentes.

Las nuevas creaciones y avances tecnológicos suponen una herramienta para el desarrollo social, el avance cultural y progreso de la ciencia. Para lograr estos avances, es necesaria la formación de profesionales competentes en el uso de herramientas digitales, para apoyar el nuevo enfoque educativo presente. Se requiere que la comunidad académica tenga presente estas necesidades para su implementación óptima en la educación superior, ya que existen docentes con poco compromiso y desventajas respecto al uso de herramientas digitales, lo cual se traduce en nulas competencias digitales. Castellanos $e$ al. [11], indican que existe un bajo dominio de las competencias digitales de profesores y estudiantes para lograr aprendizajes significativos.

De Benito-Castañedo [6], realizó un estudio que se focaliza en la brecha digital de España, la cual se ha ido complejizando con el paso de los años, al aumentar el acceso a herramientas digitales en la población. Los principales motivos de estas diferencias, son aspectos socio-culturales (etnia, edad, nivel económico y nivel académico), diferencias infraestructurales y económicas en el mundo, y falta de capital cultural en el uso de herramientas tecnológicas. También se clasifican distintos tipos de brechas digitales: de género, de edad, de índole funcional, económica, geográfica, urbana-rural, laboral, y de nivel formativo. En las brechas digitales por tema de género se indica que; en España existe un nivel inferior en formación digital de las mujeres respecto a los hombres. Si bien, esta diferencia se ha ido disminuyendo en los últimos años, todavía existe una desigualdad superior a la mayoría de los países de la UE.

Hernández y Torrijos [12], asumen como relevante considerar las exigencias establecidas para países europeos, y es por eso por lo que muchas universidades se han visto en la obligación de cambiar sus procesos educativos, centrándose en los estudiantes, e instando a los docentes a aplicar metodologías centradas en ellos, como también la incorporación de herramientas TICs en las aulas. También señalan hace falta una mayor capacitación tecnológica para incluir nuevas herramientas digitales en sus clases, pues existe una buena disposición de la comunidad educativa, pero existe un desconocimiento sobre su uso, además de existir ciertas brechas de género y edad entre los docentes.

Estudios como el realizado por Sáinz Ibáñez [13], nos muestran dos grandes diferencias en lo referente al comportamiento de hombres y mujeres, en relación con el empleo de las TICs. En primer lugar, que las mujeres participan en gran medida de las redes sociales y en menor medida de los videojuegos. Así mismo, exponen que las mujeres buscan más contenidos culturales, mientras que los hombres prefieren contenidos vinculados a juegos, deporte e informática. Las mujeres, más numerosas entre el profesorado de primaria y de secundaria, y menos numerosas en la enseñanza universitaria, tienden a percibirse menos competentes con las TICs de lo que realmente son. Esta consideración influye tanto en el uso que realizan de las TICs, como en la valoración posterior a realizar en su empleo [14, 15]. El trabajo de Sáinz Ibáñez [13] se diferencia al descrito en este artículo, en que generaliza la percepción del uso de las TIC's por géneros, mientras que nuestro trabajo, profundiza en las competencias por géneros en relación al uso y consumo de servicios específicos de internet.

Si bien el uso de nuevas tecnologías representa ventajas para los usuarios, estas también se traducen en desventajas para quienes no poseen competencias digitales. Es por esto que son necesarias 
intervenciones educativas, para capacitar a la comunidad apuntando a una mejora de los procesos de aprendizaje. Se dan algunas orientaciones para poner en práctica en el ámbito educativo, a manera de orientaciones para educadores.

Con la llegada constante de nuevos objetos tecnológicos que ya forman parte de nuestra vida diaria, se generan otros estilos de vidas y formas de relacionarse a las que debemos adaptarnos [16]. Esto da paso también a un mercado de consumo específico relacionado a las TICs, que se hace un espacio en la sociedad sin importar el nivel socioeconómico de los consumidores. Como Chandra, Ahmed y Pal [17], indican que para el consumo de un producto cobra mucha importancia el contemplarlo, comprarlo, y en el caso de la tecnología, mantenerlo en buen estado, lo cual permite una producción de nuevos espacios dedicados al consumo de tecnología. Casi todos los hogares son partícipes del consumo de nuevas tecnologías, determinados por el impacto social que estas tienen, convirtiéndose en casi una necesidad.

Los dispositivos tecnológicos están cada vez más presentes en variedad y cantidad en el espacio doméstico, con niveles de penetración que, aún siendo diferenciados, reducen las brechas digitales, redefinen lo exclusivo y las formas de vida elitizadas. Masiva incorporación de tecnologías ahorradoras de tiempo y consumidoras del mismo, que liberan espacio para el ocio posibilitando su inversión en otras actividades sociales o productivas [18].

\section{METODOLOGÍA E INSTRUMENTO}

Para esta ocasión, se trabajó en colaboración con el Instituto de Investigación en Ciencias Sociales y Educación (IICSE) de la Universidad de Atacama, a fin de poder generar nuevas experiencias en tanto visibilización de las competencias en las TICs.

La metodología llevada a cabo para este estudio corresponde a una mixta, es decir, realiza análisis descriptivo y cuantitativo. Sus etapas fueron definidas por el equipo de trabajo de esta investigación, y la cual está basada principalmente sobre análisis de datos que utilizan procesos de Minería de Datos (MD), y contiene las siguientes etapas:

1. Obtención de los datos: esta etapa permitió lograr contar con la muestra para llevar a cabo este trabajo, y que fue proporcionada el grupo de investigadores EDUINNOVAGOGiA $®$ (HUM-971).

2. Preparación de datos: en esta etapa se llevó a cabo un análisis exploratorio preliminar de los datos, en el cual se obtuvieron algunos datos estadísticos básicos. Posteriormente, se procedió a seleccionar los atributos o variables necesarias para el estudio, así como también transformar algunas variables a un tipo de dominio requerido por la técnica de análisis. También, se abordaron tareas de limpieza y depuración de datos espurios y/o faltantes. Se utilizaron en esta etapa herramientas de Extracción, Transformación y Carga de datos tales como: Data Integration de Pentaho, y el lenguaje de programación $\mathrm{R}$.

3. Selección de técnicas de análisis: En esta etapa se llevaron a cabo diferentes tareas, tales como: revisión de diferentes herramientas de análisis, tanto de MD (Weka y RapidMiner), como del lenguaje de programación $\mathrm{R}$.

4. Generación de modelos y gráficas: Para esto se ajustó la vista de datos para su análisis, según el formato de las técnicas seleccionadas para su uso, y se configuraron los parámetros mínimos de las técnicas. Con esto, se obtuvieron modelos de clústeres preliminares y finalmente se logra establecer, a través de diversas gráficas y visualizaciones, las relaciones y conglomerados representativos de los datos. Para esto se utilizó la herramienta de MD RapidMiner.

5. Validación y análisis de resultados: En esta última etapa, se procedió a realizar la validación y análisis de los resultados, para lo cual se recurre a la observación y comparación de métricas que proporcionan las técnicas. Posteriormente, se describe la interpretación de los resultados y patrones obtenidos a través de la muestra de datos.

Los datos fueron recopilados a través del cuestionario "Competencias Básicas Digitales 2.0 de estudiantes universitarios" (COBADI®, marca registrada en la Oficina Española de Patentes y Marcas: 2970648). El cuestionario COBADI ${ }^{\circledR}$ fue construido y testeado por el grupo de investigadores EDUINNOVAGOGiA $\AA$ (HUM-971), el cual es reconocido por el Plan Andaluz de Investigación, Desarrollo e Innovación y la Oficina de Transferencia de Resultados de Investigación de la Universidad Pablo de Olavide. 
El cuestionario está dividido en cinco dimensiones o bloques.

La primera dimensión, corresponde a datos relacionados con el "Consumo y uso de las TICs" en la comunicación social, trabajo individual y en grupo", compuesta por 19 ítems, y se valora de manera heterogénea a través de distintas escalas que van de 1 hasta: 2 (sí/no), 3 (nada/poco/mucho), 4, 5 o 6 puntos, estas últimas describen respuestas descriptivas. En esta dimensión prevalece la cantidad de 10 de 19 preguntas con 3 valores, y se diferencia de la demás porque trata de establecer la línea base para analizar posteriormente las competencias digitales que suponen un consumo previo de las TICs.

La segunda dimensión, corresponde a "Competencias en conocimiento y uso de las TICs en la comunicación social y aprendizaje colaborativo", compuesta por 12 ítems, y se valora a través de una escala Likert de 1-4 puntos, donde el 1 hace referencia a "yo me siento completamente ineficaz para realizar lo que se presenta" hasta el 4, que representa "yo me siento completamente eficaz". También se incluye la opción NS/NC/NA (por si el individuo desconoce la respuesta a la pregunta, o bien, si no es aplicable).

La tercera dimensión, "Competencias de uso de las TICs para la búsqueda y tratamiento de la información", la cual alude a la autopercepción de la eficacia individual en el uso de herramientas TICs. Compuesta por 12 ítems, también valorada con una escala Likert de 1-4 puntos, donde el 1 hace referencia a "yo me siento completamente ineficaz para realizar lo que se presenta" hasta el 4, que representa "yo me siento completamente eficaz", y la inclusión la opción NS/NC/NA.

La cuarta dimensión, "Competencias interpersonales en el uso de las TICs en el contexto universitario", en el cual se evalúa cómo se resuelven problemas o dudas relacionadas con las TIC. Compuesta por 5 ítems, en este bloque la escala de valoración es distinta a las dimensiones previas, donde la opción que más utilice el individuo es la primera (1), después la segunda y en último lugar la que menos use (4) para solucionar una duda.

La quinta dimensión, corresponde a "Herramientas virtuales y de comunicación social de la Universidad", compuesta por 5 ítems. Valorada con una escala
Likert de 1-4 puntos, donde el 1 hace referencia a "yo me siento completamente ineficaz para realizar lo que se presenta" hasta el 4, que representa "yo me siento completamente eficaz", y se incluye la opción NS/NC/NA.

\section{POBLACIÓN Y MUESTRA}

La muestra analizada consta de un total de 4938 estudiantes encuestados, de estos el 78,63\% son mujeres y el $21,36 \%$ hombres, la distribución por género y su alto porcentaje de mujeres, aspecto que es común en muchos estudios del área de Educación y las Ciencias Sociales, no impide el desarrollo de investigaciones sobre género [19]. Además, la muestra corresponde a estudiantes de pregrado y postgrado, por lo que su rango etario es bastante amplio, va desde los 18 hasta los 63 años de edad, teniendo como promedio de edad 22,72 años y mediana los 21 años. La distribución normal de esta variable se puede observar en el gráfico de la Figura 1, que muestra la media $(\mu)$ y cuya varianza $(\sigma 2)$ es 5,95 , y en la cual se puede verificar que la mayor cantidad de estudiantes de ambos géneros, está concentrado en el rango entre 18 y 40 años de edad.

Cabe recordar, que este estudio se basa en una muestra de datos obtenida a través de la aplicación del instrumento COBADI, el que fue respondido por un total de 4938 estudiantes. En especifico, se

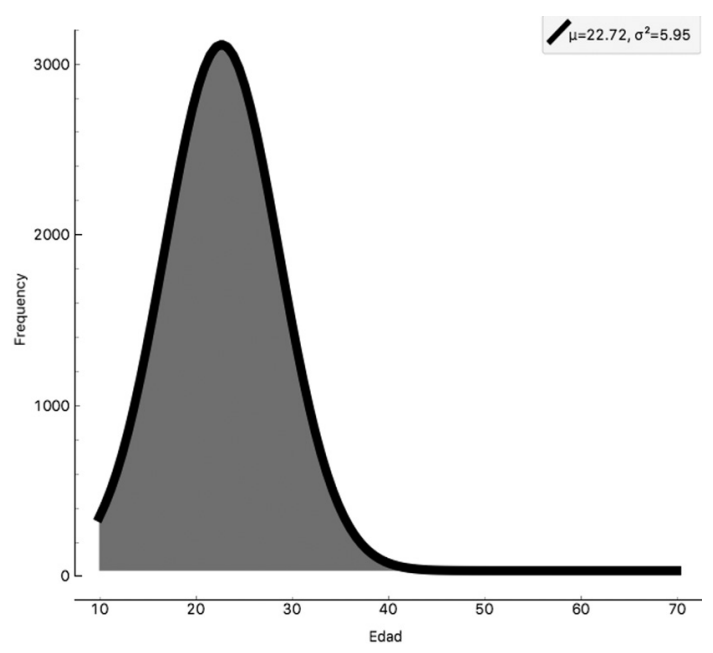

Figura 1. Distribución normal de la muestra por edad. 
considera en este análisis el resultado sólo de la primera dimensión de este instrumento denominada: “Consumo y uso de las TICs' en la comunicación social, trabajo individual y en grupo", y que tiene relación con la tenencia (disposición) de tecnología, tanto de equipamiento (computador, tablet, smartphone, etc.), así como el uso que cada estudiante le otorga a través de sus actividades personales y/o académicas. Esta dimensión contiene 19 preguntas organizadas a su vez en secciones, y que permiten observar diferentes aspectos, además que son valoradas de distinta forma, algunas preguntas con respuestas de opción múltiple (4 opciones hasta 12 en algunos casos), otras con valoración dicotómica (sí/no), y otras 10 preguntas con escala Likert de 1 a 3 (mucho/poco/nada), como se puede observar en la Tabla 1.

Adicionalmente a los datos de la dimensión señalada y descrita en la Tabla 1, se consideraron tres variables relevantes para el estudio, asociadas a la parte inicial del instrumento, y que corresponden a datos de identificación, tales como: género $(X 1)$, edad $(X 2)$, y nivel que cursa $(X 6)$.

\section{RESULTADOS}

Como resultado del proceso de análisis de los datos, conducido a través de la metodología antes descrita, y la cual se orienta en el objetivo definido para esta investigación, se comienza realizando un análisis exploratorio preliminar de los datos a través de métodos estadísticos tradicionales, y posteriormente, se utiliza una técnica de MD para generar clústeres. En particular, se utiliza la técnica de aprendizaje automático clustering (K-medias), la cual es del tipo no supervisada, y con la que se logra un análisis descriptivo de la muestra. Con esto, se logran establecer relaciones en los hábitos de uso y consumo de las TICs, por parte de los estudiantes,

Tabla 1. Dimensión utilizada del instrumento COBADI.

\begin{tabular}{|c|c|c|}
\hline \multicolumn{3}{|c|}{ Dimensión: Consumo y uso de las TICs' en la comunicación social, trabajo individual y en grupo } \\
\hline Variable & Pregunta & Valoración \\
\hline $\mathrm{X} 8$ & Has recibido formación sobre Web 2.0 o Software social? & Sí / No \\
\hline $\mathrm{X} 9 *$ & $\begin{array}{l}\text { En caso haber respondido de modo afirmativo a la anterior cuestión, por } \\
\text { favor, señale: }\end{array}$ & Opción múltiple (4 opciones) \\
\hline $\mathrm{X} 10$ & ¿Tienes ordenador? & Sí / No \\
\hline $\mathrm{X} 11$ & ¿Tienes Tablet (Ipad, Samsung Galaxi, etc.)? & Sí / No \\
\hline $\mathrm{X} 12$ & Dispones de Internet en casa. & Sí / No \\
\hline $\mathrm{X} 13$ & ¿Dónde te conectas habitualmente a Internet? & Opción múltiple (6 opciones) \\
\hline $\mathrm{X} 14$ & ¿Cuánto tiempo dedicas a navegar por Internet? & Opción múltiple (5 opciones) \\
\hline $\mathrm{X} 15^{*}$ & ¿Qué temas te interesan ver y/o buscar por Internet? & Opción múltiple (12 opciones) \\
\hline $\mathrm{X} 16^{*}$ & ¿En qué temas no perderías tu tiempo de consumo de Internet? & Opción múltiple (7 opciones) \\
\hline \multicolumn{3}{|c|}{ Puedes decirme cuánto tiempo utilizas Internet para las siguientes acciones: } \\
\hline $\mathrm{X} 17$ & Ver programas de televisión. & (nada, poco, mucho) \\
\hline $\mathrm{X} 18$ & Escuchar música. & (nada, poco, mucho) \\
\hline $\mathrm{X} 19$ & Informarse sobre temas que me interesan a nivel académico y profesional. & (nada, poco, mucho) \\
\hline $\mathrm{X} 20$ & Jugar on-line. & (nada, poco, mucho) \\
\hline $\mathrm{X} 21$ & Buscar información para realizar las tareas universitarias. & (nada, poco, mucho) \\
\hline $\mathrm{X} 22$ & Publicar fotografías/ vídeos. & (nada, poco, mucho) \\
\hline $\mathrm{X} 23$ & Bajar música, películas, juegos, etc. & (nada, poco, mucho) \\
\hline $\mathrm{X} 24$ & Hablar con los amigos a través de redes sociales, chat... & (nada, poco, mucho) \\
\hline $\mathrm{X} 25$ & $\begin{array}{l}\text { Buscar amigos/as nuevos mediante el uso de redes sociales (facebook, twitter, } \\
\text { instagam, myspace, orkut, hi5, google }+ \text { )... }\end{array}$ & (nada, poco, mucho) \\
\hline $\mathrm{X} 26$ & Trabajar en grupo con compañeros/as de clase para realizar tareas académicas)... & (nada, poco, mucho) \\
\hline
\end{tabular}

Observación: Las preguntas marcadas con * $(X 9, X 15$ y X16) fueron descartadas en este análisis, debido a que se tratan de preguntas abiertas muchas veces no contestadas. 
en labores tanto personales como en sus actividades universitarias, de modo tal de verificar la diferencia existente entre géneros y la edad.

Un primer análisis señala la composición de la muestra en relación a las variables: género, curso y edad, lo cual se puede observar a partir del gráfico de la Figura 2.

Este gráfico ratifica la población mayor de mujeres y la distribución normal descrita en el gráfico de la Figura 1, además que en los primeros 4 cursos sus programas de estudios, el 75,42\% corresponden a mujeres (3724) y el 20,37\% a hombres (1006) respecto del total de la muestra, lo que corresponde para ambos géneros, aproximadamente el $95 \%$ del subtotal por género.

El análisis posterior se realizó en base a la estructura de las preguntas y su forma de ser valorada, para tener una mejor aproximación. Es por esto, que se agruparon en tres secciones:

1. La primera sección; con las preguntas $X 8$, $X 10, X 11$, y $X 12$ por su forma dicotómica (Sí / No) para ser valorada. Que se refieren fundamentalmente, a la disposición o tenencia de la tecnología necesaria para conectarse a Internet.

2. La segunda sección; con las preguntas $X 13$ y X14 por tener respuestas con opción múltiple y acotadas. Estas preguntas apuntan al lugar y tiempo de conexión a Internet.

3. Y, la tercera sección; con las preguntas $X 17$ a las $X 26$, por contar con valoración a través de escala Likert de 3 niveles (mucho, poco, nada). Las preguntas de esta sección, apuntan a conocer el tiempo de consumo de la tecnología (aplicaciones/servicios) Internet en distintas actividades cotidianas, tanto de ocio como académicas.

Respecto sobre la tenencia de tecnología, se pueden observar lo siguientes resultados en la primera sección:

- $\quad$ El 71,7\% de los encuestados no recibieron formación sobre Web2.0 o Software social (X8).

- El 96\% de los encuestados cuenta con un computador $(X 10)$

- $\quad$ El 54,1\% de los encuestados posee una Tablet (X11).

- $\quad$ El 94,3\% de los encuestados cuenta con conexión a internet (X12).

En cuanto al lugar y tiempo de conexión a Internet, se observan los siguientes resultados en la segunda sección de la encuesta:

- En relación a donde se conectan los encuestados a internet, el 50,6\% se conecta desde casa y el $43,6 \%$ se conecta desde cualquier lugar ya que cuentan con internet móvil (X13).

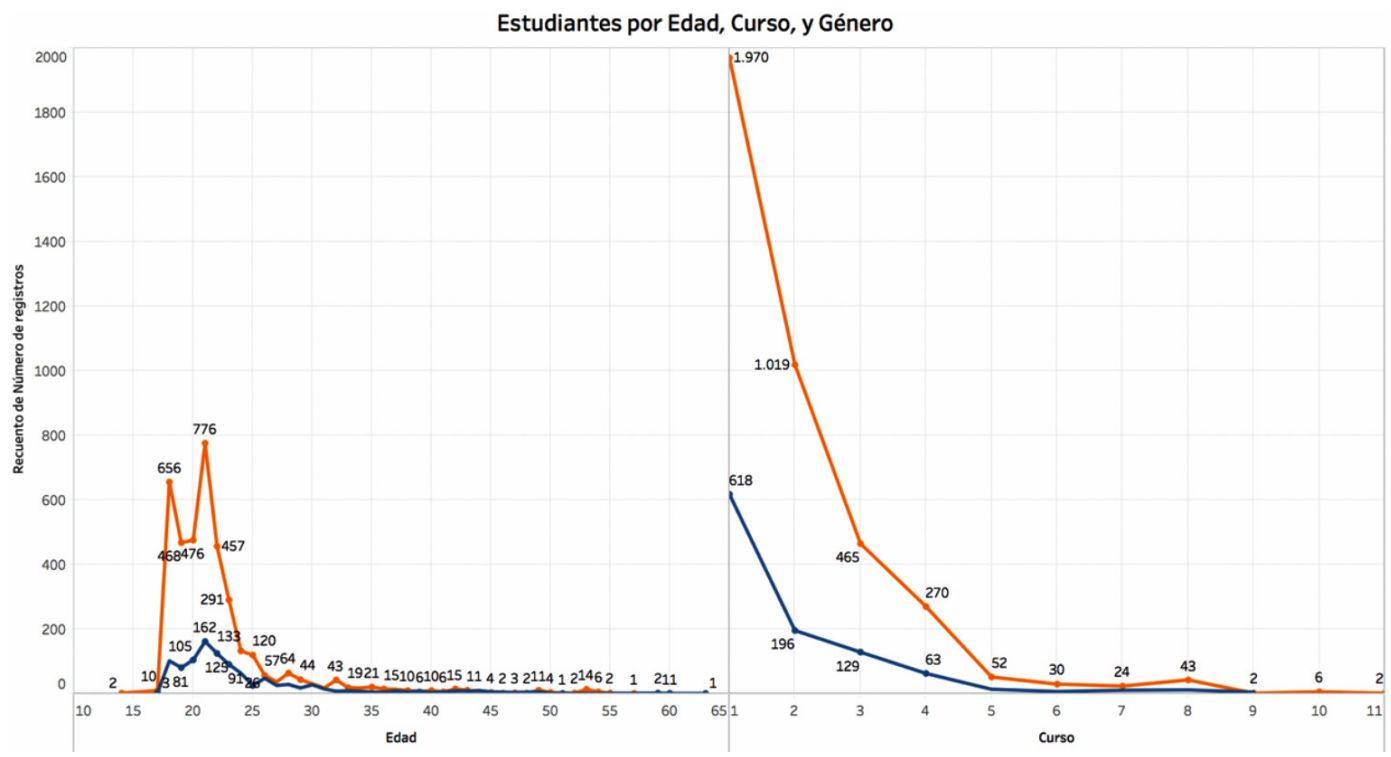

Figura 2. Cantidad de estudiantes por Edad, Curso y Género. 
- Además, se observa que el 18,36\%, 31,15\%, y $48,59 \%$ de los encuestados se conectan a internet entre 1 y 3 horas, 4 y 9 horas, y más de 9 horas semanales respectivamente (X14).

Finalmente, en lo que se refiere al consumo de la tecnología a través de las distintas aplicaciones o servicios de Internet, se obtienen los siguientes resultados en la tercera sección:

- El 82,8\% de los encuestados, ven poco y nada de televisión por internet mientras que el 17,2\% no ve nada de televisión a través de internet (X17).

- El 68,5\% de los encuestados escuchan mucha música a través de internet mientras que el $31,5 \%$ escuchan poco y nada de música por internet $(X 18)$.

- El 64,6\% utilizan mucho la internet para informarse sobre temas académicos y profesional, mientras que el $33,73 \%$ lo utilizan poco y solo un $1,67 \%$ no utilizan internet para fines académicos y profesional (X19).

- Respecto al uso de la tecnología para juegos on-line, se observa que el $73,61 \%$ de los encuestados no juegan videojuegos on-line, un $19.8 \%$ lo utilizan poco, y un $6,59 \%$ lo utilizan mucho $(X 20)$.

- En buscar información para tareas universitarias, un $82,6 \%$ de los encuestados utilizan mucho la internet para este fin, mientras que solo un
$16,77 \%$ lo utilizan poco y un $0,63 \%$ no utilizan en absoluto la internet para estos fines (X21).

- Para publicar fotografías/videos en internet, un $50,78 \%$ lo utilizan poco, un $34,83 \%$ lo utiliza mucho y solo un $14,39 \%$ no lo utiliza en absoluto (X22).

- Para bajar música, películas, juegos, etc., se observa que un $23,35 \%, 43,12 \%$, y un $33,53 \%$ utilizan respectivamente nada, poco y mucho la internet para estos fines (X23).

- En lo que refiere a hablar con los amigos a través de redes sociales, se observa que un $72,8 \%$ utilizan mucho la internet para este fin y solo un $23,09 \%$, y $4,11 \%$ utilizan poco y nada respectivamente la internet para este fin $(X 24)$.

- En buscar amigos a través de redes sociales, el $81,2 \%$ de los encuestados utilizan poco y nada las redes sociales, y solo un $18,8 \%$ lo utilizan para este fin $(X 25)$.

- Finalmente, respecto a trabajar en grupo con compañeros de clase para realizar actividades académicas, el 59\% utiliza mucho la internet para este fin, y un $4,83 \%$, y $36,17 \%$ de los encuestados utilizan respectivamente nada y poco la internet para este fin $(X 26)$.

\section{ANÁLISIS Y DISCUSIÓN}

En general, la mayoría de los estudiantes encuestados cuentan con las tecnologías necesarias para acceder

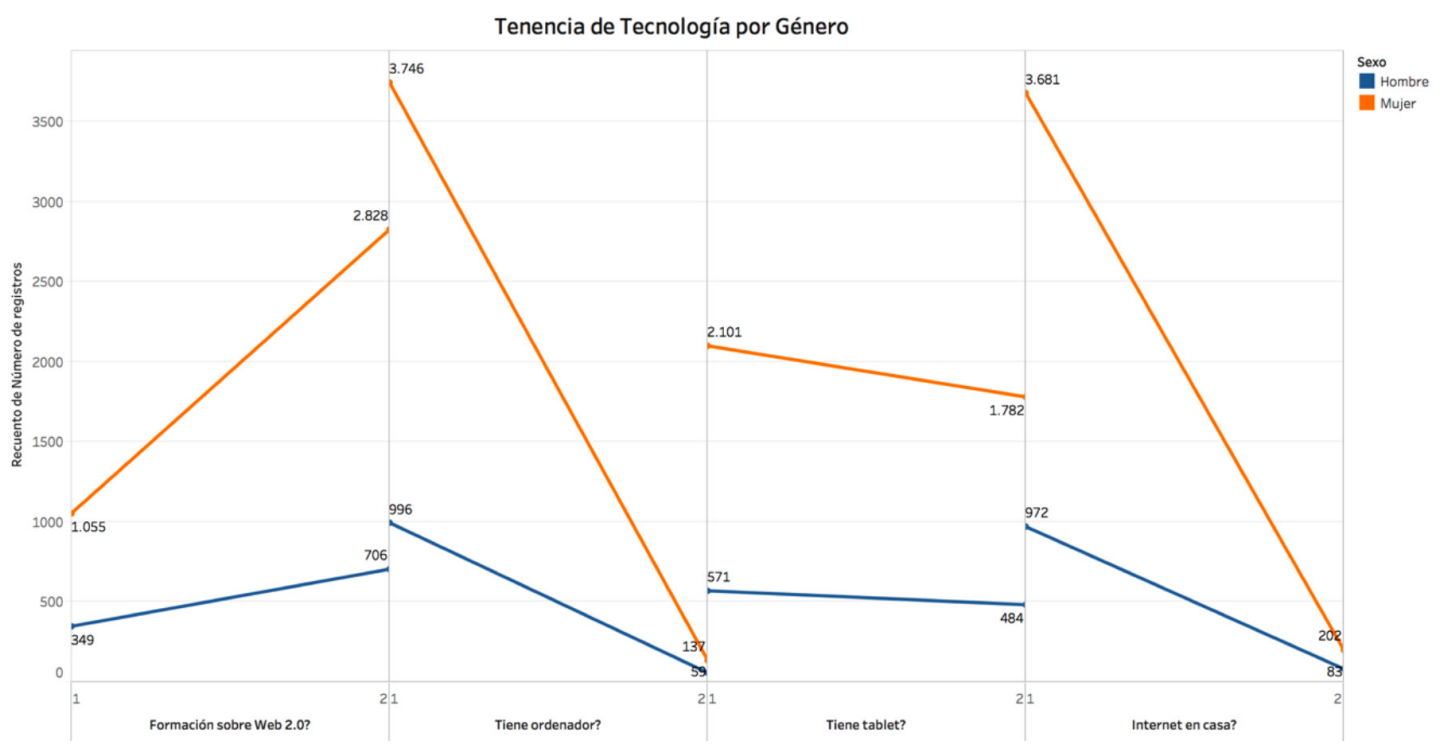

Figura 3. Sección 1 de la dimensión analizada. 
a internet. Un análisis comparativo respecto al género, permite observar diferencias de sus hábitos de consumo en las tres secciones de la encuesta, así como en el uso algunas aplicaciones y/o servicios de Internet.

Es así como el gráfico de la Figura 3, a continuación, muestra el comportamiento de la primera sección, y que tiene relación a la disposición o tenencia de las TICs.

En este gráfico se observan las 4 variables ( $X 8$, $X 10, X 11, y X 12)$, descritas con las leyendas en el eje $\mathrm{x}$, que han sido descritas en la Tabla 1, y cuyas respuestas son dicotómicas, donde "Sí" corresponde a 1, y "No" al valor 2 de este eje. Se puede apreciar en esta Figura 3, un resultado similar para ambos géneros respecto a las 4 preguntas. Solo se ve una pequeña diferencia en la primera pregunta 1 , con un $73 \%$ de las mujeres que responden no haber recibido formación sobre Web 2.0, y los hombres responden también negativamente con un $67 \%$.

La segunda sección agrupada de la dimensión analizada, y que contiene solo 2 preguntas, cuyas respuestas corresponden al tipo opción múltiple acotado, que tienen relación con la conexión a Internet, donde los estudiantes se conectan (X13) y cuanto tiempo lo hacen $(X 14)$, esto lo presenta gráficamente la siguiente Figura 4.
En el análisis por género, destaca en este gráfico de la Figura 4 que, en proporción, las mujeres son las que más utilizan el móvil como lugar de conexión a Internet con aproximadamente un $46 \%$ del total, respecto a los hombres que sólo llegan a un 35\% aproximadamente y que declaran en mayor medida la preferencia de conectarse en su hogar con cerca de un 59\%, a diferencia de las mujeres que lo hacen con un $48 \%$. También son las mujeres que, según los resultados, pasan más tiempo conectadas a Internet para distintas actividades, cerca de un 50\% sobre 9 horas a la semana, siendo casi 7 puntos menos los hombres con un $43 \%$ aproximadamente.

Finalmente, la tercera sección que agrupa las preguntas $X 17$ hasta la pregunta $X 26$, y que se orientan específicamente al uso y consumo de aplicaciones y/o servicios a través de Internet, cuyas respuestas son valoradas a través de una escala Likert de 3 niveles donde: nada es representada con el valor 1 , poco con valor 2 y mucho con el valor 3 . Esto permite analizar y comparar los hábitos de este consumo a través del género, lo cual se puede ver en la Figura 5.

El análisis por género, permite observar que las mujeres tienden a utilizar en mayor medida casi todos los servicios Internet, en esta muestra en 9 de las 10 preguntas de esta sección, presentan aproximadamente un $80 \%$ como respuesta utilizar "mucho" cada servicio consultado, y solo en la pregunta $X 20$ (juego on-line) son superadas con las

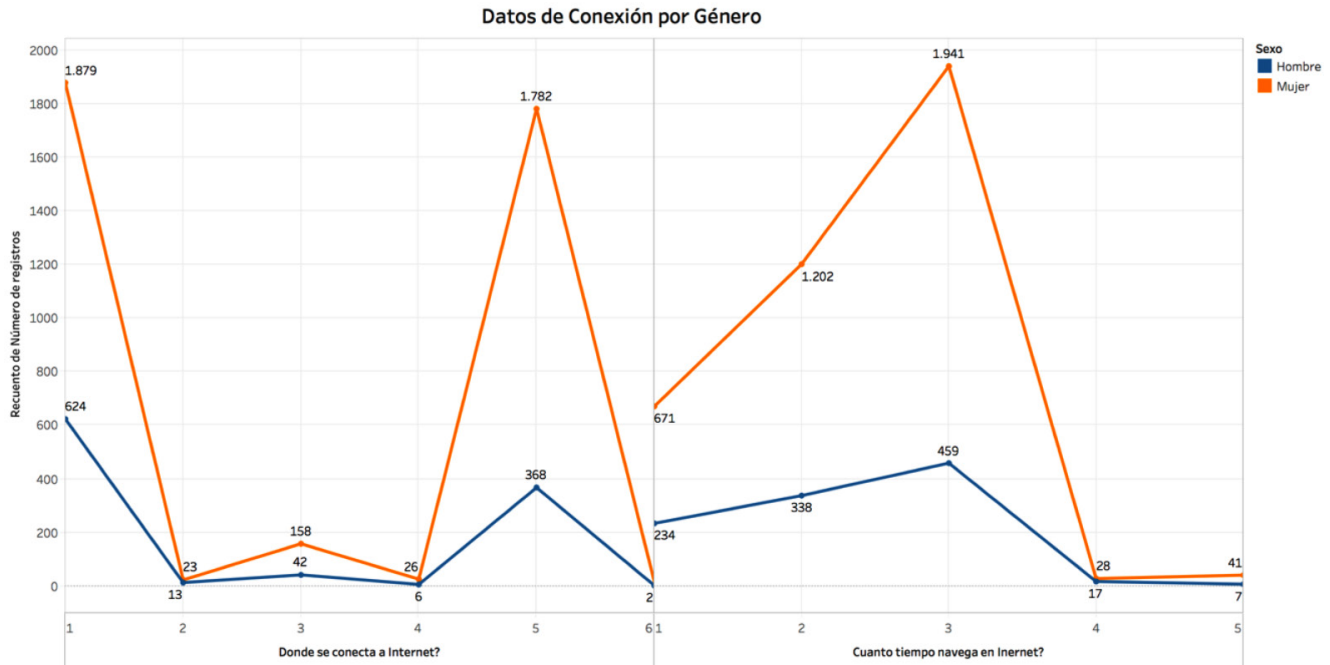

Figura 4. Sección 2 de la dimensión analizada. 


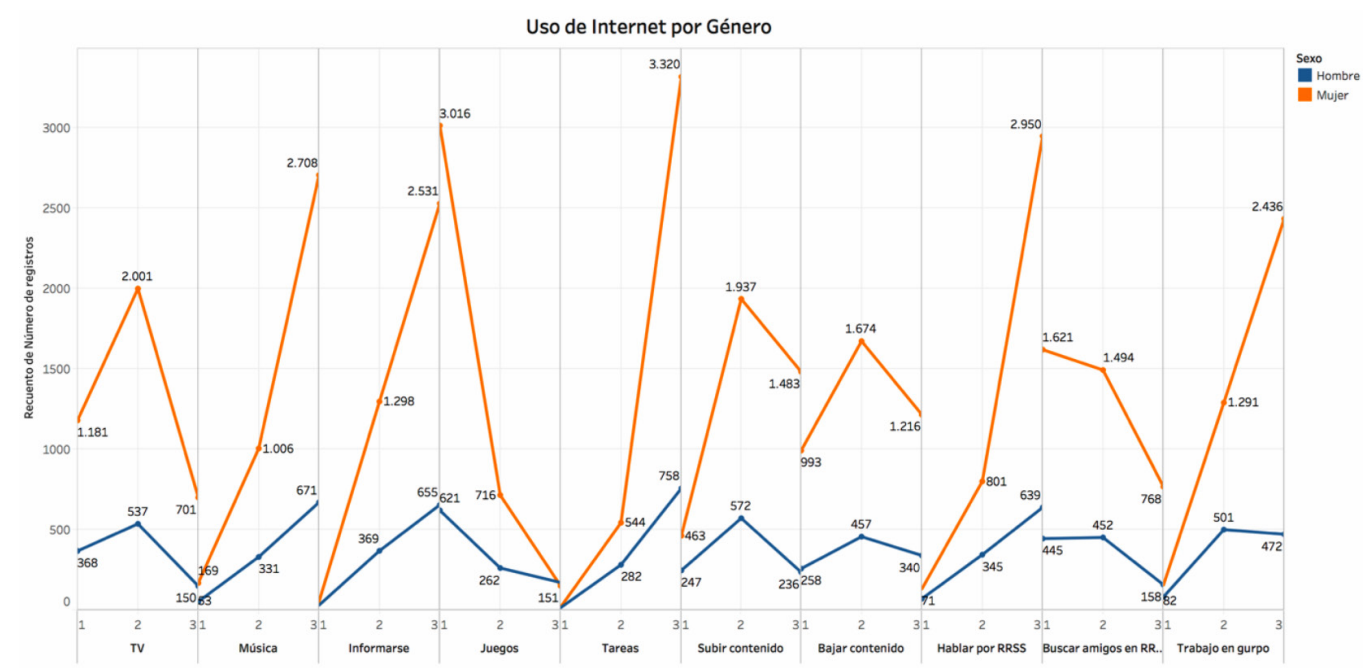

Figura 5. Sección 3 de la dimensión analizada.

respuestas de los varones en proporción porcentual, en la cual declaran en un $82 \%$ no utilizar en "nada" Internet para jugar. Desde una perspectiva general de cada una de las variables de esta sección, se puede ver en el gráfico de la Figura 6, el detalle de cada una.

Destaca en este análisis, el hecho que las mujeres sobresalen en el uso de las TICs para labores de búsqueda de información para realizar tareas universitarias $(X 21)$ con un $82 \%$, así como en el uso para subir contenidos (fotografías/videos $X 22$ ) con un $86 \%$, y también el utilizar los servicios de redes sociales para comunicarse con amigos $(X 24)$ con un $82 \%$ respecto del total.
Por otro lado, los hombres solo tienen valores proporcionales sobre el $60 \%$ en los ítems X18, X19, $X 21$ y $X 24$. Esto puede ser observado a partir de la Figura 6, en la que además se presenta no solo el detalle acumulado de las respuestas en cada una de las variables, sino que además la tendencia medida en promedio por género. El detalle de los valores obtenidos en este análisis se encuentra en la Tabla 2:

En cuanto al proceso de análisis a través de $\mathrm{MD}$, con la técnica de clustering K-medias, se generaron modelos con distinta cantidad de clústeres $(\mathrm{k})$, tratando de lograr describir de mejor manera los hábitos de uso y consumo de servicios Internet diferenciando por género. Se utilizó para realizar

\section{USO Y CONSUMO DE SERVICIOS INTERNET}

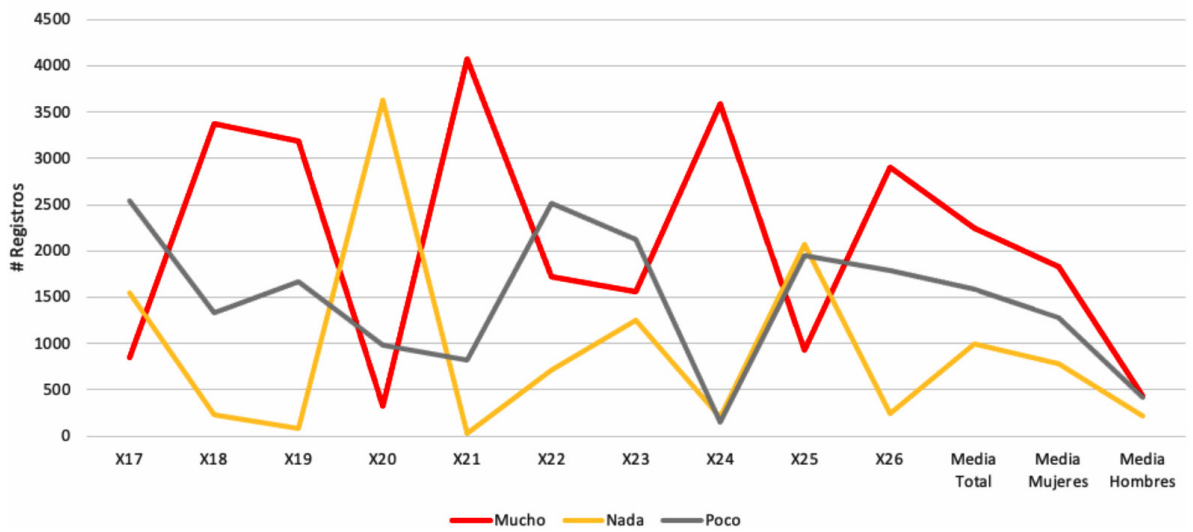

Figura 6. Visión general de la tercera sección. 
Tabla 2. Valorizaciones totales obtenidas en la sección de la dimensión analizada.

\begin{tabular}{|c|c|c|c|c|c|c|c|c|c|c|c|c|c|c|c|c|}
\hline Respuesta & $\mathrm{X} 17$ & $\mathrm{X} 18$ & X19 & $\mathbf{X} 20$ & $\mathbf{X} 21$ & $\times 22$ & $\times 23$ & $\times 24$ & X25 & $\times 26$ & \multicolumn{2}{|c|}{ Media Total } & \multicolumn{4}{|c|}{ Media Mujeres Media Hombres } \\
\hline Mucho & 851 & 3379 & 3186 & 323 & 4078 & 1719 & 1556 & 3589 & 926 & 2908 & 2252 & $46,5 \%$ & 1826 & $47,0 \%$ & 425 & 40,3 \\
\hline Nada & 1549 & 222 & 85 & 3637 & 34 & 710 & 1251 & 203 & 2066 & 238 & 1000 & $20,7 \%$ & 780 & $20,1 \%$ & 219 & $20,8 \%$ \\
\hline Poco & 2538 & 1337 & 1667 & 978 & 826 & 2509 & 2131 & 146 & 1946 & 1792 & 1587 & $32,8 \%$ & 1276 & $32,9 \%$ & 411 & 38,9 \\
\hline
\end{tabular}

este proceso de MD, la herramienta RapidMiner Studio versión 9.7.

Como se puede observar en la Figura 7, para configurar el proceso de $\mathrm{MD}$, se seleccionaron todas las variables descritas en la Tabla 1 excepto la variable $X 13$, esto debido a que representa una pregunta cuya respuesta es del tipo opción múltiple con 6 distintas opciones, cada una con un peso irrelevante en el conjunto de datos y difícil de lograr una adecuada normalización. Luego, se procedieron a normalizar todas las variables seleccionadas (nodo Normalize), de modo que puedan quedar en una escala similar, la cual fue el rango $[-1.0,+1.0]$.

Con esto se logra facilitar el proceso de agrupamiento de la técnica K-medias, ya que hay que recordar que los clústeres se forman a partir del cálculo de las distancias, y al existir variables con escalas muy diferentes, como es el caso del conjunto de datos analizado, las variables de mayor escala dominarían las distancias. Finalmente, se ejecutan de manera simultánea los cuatro nodos de la técnica K-medias para generar los modelos con valores diferentes para el número de clústeres $(k=2,3,4$ y 5$)$. De los modelos obtenidos en este proceso, se selecciona aquel con el número de clústeres $k=2$, cuyo resultado se presenta en la Figura 8. Esta selección se debió principalmente, por un lado, porque permite diferenciar claramente el comportamiento de la muestra por género, lo que está alineado al objetivo del estudio, y por otro lado, porque logra validar en gran parte, lo explorado previamente a través de los métodos estadísticos.

En la gráfica de la Figura 8, la línea de color azul representa al clúster cero (0), y la de color rojo al clúster uno (1). En el eje $x$ están dispuestas todas las variables (excepto X13), en el eje $y$ se dispone la escala normalizada en el rango descrito anteriormente. La interpretación de los clústeres es la siguiente:

- Clúster 0 (azul): 3483 instancias, mayoritariamente corresponden a mujeres, promedio de edad 22 años, más del $90 \%$ entre los cursos 1 al 4 , en general navegan más de entre 4 y 9 horas a la semana (X14), utilizan mucho Internet para: escuchar música (X18), publicar fotografías/ vídeos $(X 22)$, y hablar con los amigos a través de redes sociales $(X 24)$.

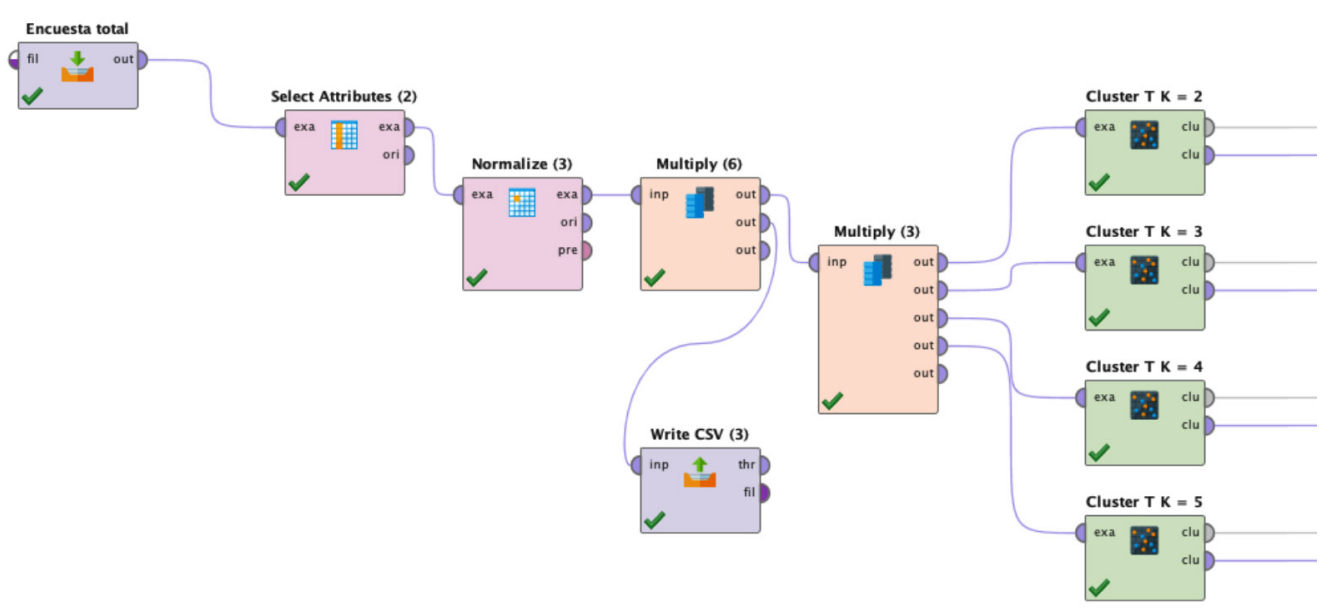

Figura 7. Proceso de MD. 
- Clúster 1 (rojo): 1455 instancias, mayoritariamente corresponden a varones, promedio de edad 25 años, más del $95 \%$ entre los cursos 1 al 5 , en general navegan más de 9 horas a la semana (X14), utilizan poco Internet para: escuchar música (X18), publicar fotografías/vídeos $(X 22)$, y hablar con los amigos a través de redes sociales $(X 24)$.

De esta interpretación, se puede apreciar claramente la diferencia entre ambos géneros en relación a los hábitos de uso y consumo de servicios Internet, en particular esto se acentúa visualmente en la gráfica de la Figura 8, a partir de la separación mayor de las líneas de color rojo y azul, entre las variables $X 20$ a $X 26$. Solo coinciden, en el uso de estos servicios para informarse sobre temas relacionados con sus labores académicas y profesionales en aproximadamente un $63.5 \%$, lo que se ve reflejado en la intersección de las líneas en la variable $X 19$. Este análisis, corrobora en gran medida el análisis exploratorio preliminar que fue realizado utilizando métodos estadísticos.

\section{CONCLUSIONES Y TRABAJO FUTURO}

El trabajo realizado en esta investigación se centró en el análisis de los datos obtenidos a través de la aplicación del instrumento COBADI, y sobre el que se pudo lograr contar con una muestra de 4938 respuestas de estudiantes universitarios, en su mayoría mujeres $(78,63 \%)$, esto se debe porque se trata de una facultad del área de las humanidades, en cuyas carreras es de mayor interés en este género. El objetivo a lograr fue obtener una clasificación del uso de las TICs por género, que permita reconocer y confirmar los hábitos de uso y consumo de los servicios digitales a través de Internet. El trabajo se realizó utilizando una metodología mixta, es decir desde un enfoque descriptivo se obtuvieron resultados cuantitativos para explicar los hábitos de los estudiantes respecto al objetivo definido.

En primer lugar, los dos géneros declaran contar con los medios tecnológicos necesarios para utilizar los servicios de Internet, en aproximadamente un $95 \%$. Eso sí, los resultados demuestran que existe una clara diferenciación de los propósitos que focalizan el uso de las TICs según la perspectiva de género, por un lado, en las mujeres prevalece la ubicuidad tecnológica, esto es, estar siempre conectada a Internet a través de su móvil, con respecto a los hombres que se conectan mayormente desde el hogar. Además, también son las mujeres (50\%) las que pasan mayor tiempo conectadas a la red en relación con los hombres (43\%).

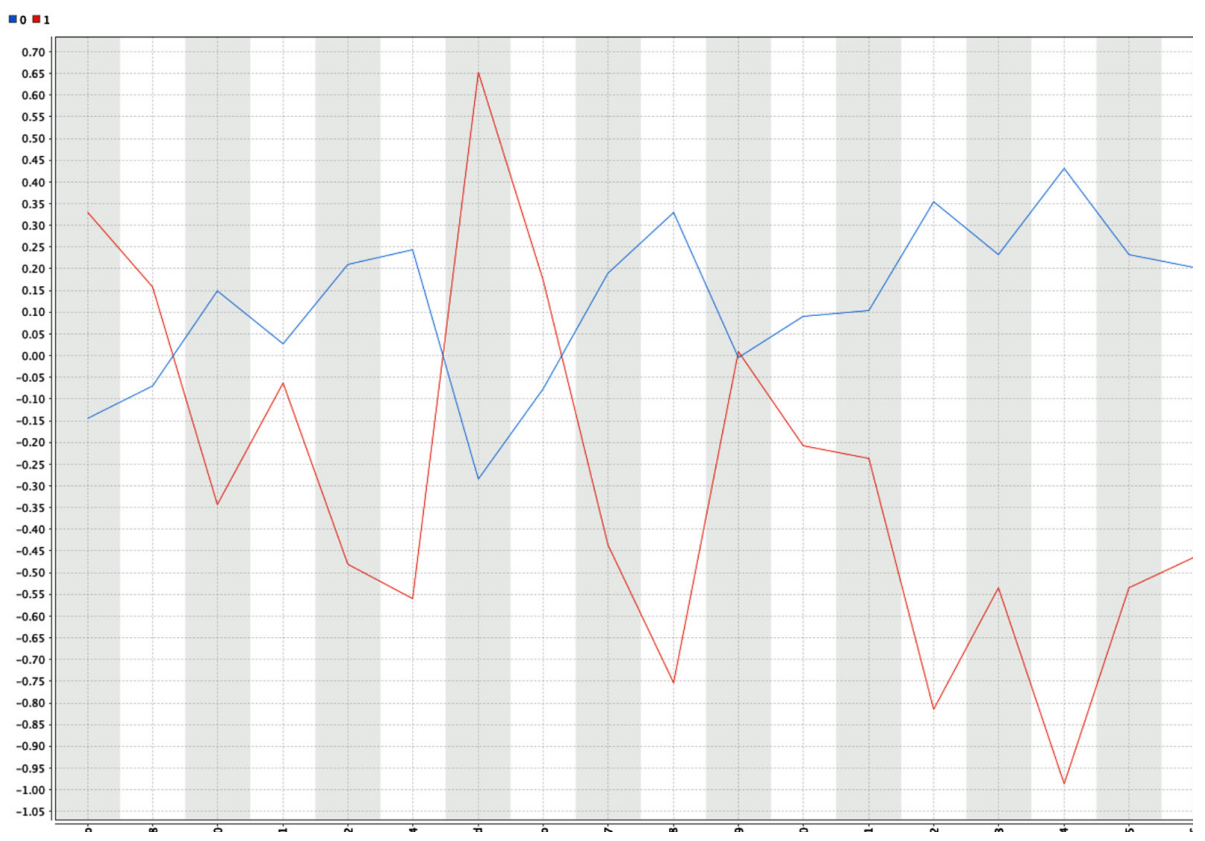

Figura 8. Visualización del modelo clustering seleccionado. 
Lo anterior, se ve reforzado con el análisis de la última sección, donde las mujeres priorizan el uso de los servicios o aplicaciones de internet como redes sociales para comunicarse no tan solo a nivel personal, sino que, para realizar trabajos universitarios, lo cual se logra trazar las variables $X 21$ con la X24 ambas con una valoración del 82\%, muy lejos de los hombres. Así como también, son las mujeres que más utilizan los servicios digitales para subir contenidos $(X 22)$ con un $86 \%$ versus un $13.7 \%$ de los hombres.

Como trabajo futuro se consideran que:

- Los resultados de este trabajo permiten obtener una línea base, para profundizar un análisis enfocado a determinar el nivel de competencias digitales desarrolladas por los estudiantes, y desde una perspectiva de género en un trabajo posterior.

- También el trabajo logra establecer una clasificación y diferenciación por género del uso y consumo de algunos servicios tecnológicos.

- Se considera profundizar este estudio, aumentando la cantidad de registros de la muestra para ser analizada y que sea homogénea en cantidad por género.

- Además, se tienen considerado incorporar en el proceso de análisis, herramientas descriptivas adicionales. Desde esta perspectiva, lograr generar un modelo de MD que permita visualizar con mayor profundidad las brechas de las competencias digitales.

\section{REFERENCIAS}

[1] A. Serrano, A. Biedermann y J.L. Sáenz. "Objetivos, competencias y expectativas de futuro profesional de los estudiantes del grado en ingeniería en diseño industrial y desarrollo de producto". REDU. Revista de Docencia Universitaria. Vol. 14 No 1, pp. 69-96. 2016. DOI:10.4995/redu.2016.5908.

[2] United Nations Educational, Scientific and Cultural Organizationn UNESCO. "ICT competency framework for teachers". UNESCO. 2019.

[3] M. Marza y E. Cruz. "Gaming como instrumento educativo para una educación en competencias digitales desde los academic skills centres". Revista General de Información y Documentación. Vol. 28
$\mathrm{N}^{\mathrm{o}} 2$, pp. 489-506. 2018. DOI: 10.5209/ RGID.60805.

[4] D. Fernández Zalazar. Usos y apropiación de las TIC en función de las competencias digitales en la construcción de conocimiento de los estudiantes de psicología de la universidad de Buenos Aires". XI Congreso Internacional de Investigación y Práctica Profesional en Psicología. Universidad de Buenos Aires. 2019. Buenos Aires, Argentina.

[5] L. Lévano-Francia, S. Sanchez, P. GuillénAparicio, S. Tello-Cabello, N. Herrera-Paico y Z. Collantes-Inga. "Competencias digitales y educación: Propósitos y representaciones". Vol. $7 \mathrm{~N}^{\circ}$ 2, pp. 569-588. 2019. DOI: 10.20511/pyr2019.v7n2.329.

[6] J. De Benito-Castanedo. "Bibliographic analysis of the digital divide and literacy in new technologies". Revista Electrónica Educare. Vol. $21 \mathrm{~N}^{\circ}$ 2, pp. 1-10. 2017. DOI: 10.15359/ree.21-2.9.

[7] G.Y. Calle y A. Lozano. "La alfabetización digital en la formación de competencias ciudadanas en la básica primaria”. Revista Eleuthera. Vol. 20, pp. 35-54. 2018. DOI: 10.17151/eleu.2019.20.3.

[8] OCDE. "How's life in the digital age?: Opportunities and risks of the digital transformation for people's well-being". OECD Publishing. 2019. París, Francia.

[9] Parlamento Europeo y el Consejo de la Unión Europea. "Recomendación del parlamento europeo y del consejo, de 18 de diciembre de 2006, sobre las competencias clave para el aprendizaje permanente". Diario Oficial L 394 de 30.12.2006. http://data.europa.eu/ eli/reco/2006/962/oj. 2006.

[10] J. Gutiérrez, J. Guzmán y Y. Jiménez. "Aplicación de las competencias digitales docentes en el uso del entorno b-learning de la escuela superior de computo del instituto politécnico nacional. Reflexiones sobre la equidad de género". Edunovatic 2016. Congreso Virtual internacional de Educación, Innovación y TIC. Libro de actas, pp. 621629. 2016.

[11] M. Castellanos, Z. Nieto Sánchez y H. Parra López. "Interpretación de las competencias digitales profesorales presentes en el contexto universitario". Revista Logos, Ciencia \& 
Tecnología. Vol. $10 \mathrm{~N}^{\mathrm{o}}$ 1, pp. 41-51. 2018. ISSN: 2145-549X.

[12] J.P. Hernández Ramos y P. Torrijos Fincias. "Percepción del profesorado sobre la integración de las tecnologías de la información y la comunicación (TIC) en las modalidades docentes, Influencia del género y la edad". EDMETIC, Revista de Educación Mediática y TIC. Vol. $8 \mathrm{~N}^{\circ} 1$, pp. 128-146. 2018. DOI: 10.21071/edmetic. v8i1. 10537.

[13] M. Sainz Ibañez. "El uso de las TIC en el ámbito educativo con perspectiva de género. Actitudes del profesorado y del alumnado". Telos: Cuadernos de comunicación e innovación. Vol. 95, pp. 116-124. 2013.

[14] A. García-Valcarel y F. Tejefor. "Variables TIC vinculadas a la generación de nuevos escenarios de aprendizaje en la enseñanza universitaria". Aportes de las Curvas ROC para el análisis de diferencias. Educación XXI: Revista de la Facultad de Educación. Vol. $14 \mathrm{~N}^{\circ} 12$, pp. 43-78. 2011. DOI: 10.5944/ educxx1.14.2.237.

[15] M. Sáinz and M. López-Sáez. "Gender differences in computer attitudes and the choice of technology-related occupations in a sample of secondary students in Spain".
Computers \& Education. Vol. $54 \mathrm{~N}^{\mathrm{o}} 2$, pp. 578-587. 2010. DOI: 10.1016/J. COMPEDU. 2009.09.007.

[16] S. Cruces, R. Guil, N. Sánchez y J. Pereira. "Consumo de nuevas tecnologías y factores de personalidad en estudiantes universitarios". Revista de Comunicación y Ciudadanía Digital. Vol. 5 No 2, pp. 203-228. 2016.

[17] P. Chandra, S. Ahmed and J. Pal. "Market practices and the bazaar: Technology consumption in ICT markets in the global south". In Proceedings of the 2017 CHI Conference on Human Factors in Computing Systems (CHI'17). Association for Computing Machinery. 2017. DOI: 10.1145/3025453.3025970.

[18] C. Rodríguez y D. Sandoval. "Consumo tecnológico: Análisis de los determinantes del equipamiento doméstico mediante arboles de decisión. Revista Internacional de Investigación en Ciencias Sociales", pp. 70-85. 2015. DOI: 10.18004/riics.2015. julio.70-85.

[19] V. Gialamas, K. Nikolopouiou and G. Kutromanos. "Student teachers? perceptions about the impact of internet usage on their learning and jobs". Computers \& Education. Vol. 62, pp. 1-7. 2013. 